\title{
Incorporation of the Endoplasmic Reticulum Stress-Induced Spliced Form of XBP1 mRNA in the Exosomes
}

\author{
Toru Hosoi*, Mieko Nakashima and Koichiro Ozawa* \\ Department of Pharmacotherapy, Graduate School of Biomedical and Health Sciences, Hiroshima University, Hiroshima, \\ Japan
}

It is known that endoplasmic reticulum (ER) and nucleus communicate with each other to cope with ER stress. However, the mechanisms through which extracellular transmission of ER stress occurs remain unexplored. When the ER stress-induced unfolded protein response (UPR) is activated, the X-box binding protein 1 (XBP1)

OPEN ACCESS

Edited by:

Guido Santos-Rosales, Universitätsklinikum Erlangen,

Germany

Reviewed by:

José Javier López Barba, Universidad de Extremadura, Spain Bredford D. Kerr Centro de Estudios Científicos, Chile Carlos Puebla

Universidad Autónoma de Chile, Chile

*Correspondence: Toru Hosol toruh@hiroshima-u.ac.jp Koichiro Ozawa ozawak@hiroshima-u.ac.jp

Specialty section: This article was submitted to Membrane Physiology and Membrane Biophysics, a section of the journal

Frontiers in Physiology

Received: 11 May 2018 Accepted: 07 September 2018 Published: 26 September 2018

Citation:

Hosoi T, Nakashima M and Ozawa K (2018) Incorporation of the Endoplasmic Reticulum Stress-Induced Spliced Form of XBP1 MRNA in the Exosomes. Front. Physiol. 9:1357. doi: 10.3389/fphys.2018.01357 mRNA is spliced by inositol-requiring enzyme-1 $\alpha$ (IRE1 $\alpha)$ to produce the spliced form of XBP1 (sXBP1). In the present study, we found that sXBP1 mRNA in the cell may be incorporated into the exosomes and was released extracellularly. We found that the ratio of the mRNA levels of SXBP1 to unspliced XBP1 (UXBP1) in the exosome was higher than that of cells in MIN6 mouse pancreatic $\beta$ cells. A similar effect was observed when XBP1 splicing was induced by overexpressing IRE1 $\alpha$ in HEK293T cells. These results suggest that the incorporation of sXBP1 into the exosomes is a novel mechanism of UPR transmitted to extracellularly, which would be triggered when cells are exposed to stress.

Keywords: exosome, unfolded protein response, endoplasmic reticulum stress, IRE1 $\alpha$, XBP1

\section{INTRODUCTION}

Cells encounter various environmental stress stimuli, which perturb the functioning of endoplasmic reticulum (ER). Such stimuli cause accumulation of unfolded proteins in the ER, which in turn induces the cells to activate the unfolded protein response (UPR) to cope with such a stress (ER stress) (Walter and Ron, 2011). To date, three major types of UPR stress transducer proteins have been identified: inositol-requiring enzyme-1 (IRE1), double stranded RNA-activated protein kinase (PKR)-like ER kinase (PERK), and activating transcription factor 6 (ATF6) (Ron and Walter, 2007). Activation of these stress transducer proteins conveys the stress signals to the nucleus. Activation of IRE1 triggers X-box binding protein 1 (XBP1) mRNA splicing, and the spliced form (sXBP1) enters the nucleus and acts as a transcription factor (Yoshida et al., 2001; Calfon et al., 2002).

Mounting evidence has shown that the ER and nucleus communicate with each other to cope with ER stress (Hetz, 2012). However, as far as we know, there is no previous evidence showing the extracellular transmission of ER stress through exosome. The exosomes are nanoscale vesicles (50$150 \mathrm{~nm}$ in diameter) that are secreted extracellularly. Increasing evidence suggests that the secreted exosomes play a role in cell-cell communication (Valadi et al., 2007; Schorey et al., 2015). In the present study, we hypothesized that the ER stress signals may be transduced and packed into the exosomes. 


\section{MATERIALS AND METHODS}

\section{Materials and Reagents}

Tunicamycin was obtained from Wako Pure Chemical Industries, Ltd. (Japan). ExoQuick-TC was obtained from System Biosciences (CA). Exosome-depleted FCS was obtained from System Biosciences (CA) or Life Technologies (CA). We wear gloves and lab coat and used hoods for the preparation of biohazard elements.

\section{Cell Culture}

HEK293T cell line was cultured in DMEM with $10 \%$ fetal calf serum (FCS), 100 units/ml of penicillin G, $100 \mu \mathrm{g} / \mathrm{mL}$ of streptomycin, and $0.25 \mu \mathrm{g} / \mathrm{mL}$ of amphotericin B (Nacalai Tesque Inc., Japan). The MIN6 cell line was cultured in DMEM with $50 \mu \mathrm{M} \beta$-mercaptoethanol, 10\% FCS, 100 units $/ \mathrm{mL}$ of penicillin $\mathrm{G}, 100 \mu \mathrm{g} / \mathrm{mL}$ of streptomycin, and $0.25 \mu \mathrm{g} / \mathrm{mL}$ of amphotericin B (Nacalai Tesque Inc.). The cells were cultured at $37^{\circ} \mathrm{C}$ with $5 \% \mathrm{CO}_{2}$.

\section{Transfection}

On the starting day (day 0), the HEK293T cells were plated onto $100-\mathrm{mm}$ dishes. On day 1 , the cultured cells were replenished with fresh medium (DMEM, 10\% FCS) not containing antibiotics. Three hours later, the cells were transfected with IRE1 $\alpha$ (FLAG-tagged human IRE1 $\alpha$ ) plasmid by using the calcium phosphate co-precipitation method. Sixteen hours after the transfection, the cells were replenished with fresh medium (DMEM), which included exosome-depleted FCS (5\%) and antibiotics.

\section{RT-PCR}

Total RNA was isolated and RT-PCR was performed as previously described, albeit with a few modifications (Hosoi et al., 2016). For PCR amplification, we used the Expand High Fidelity kit (Roche AG) and KAPA Taq Extra PCR kit (KAPA Biosystems). The following primers were used: $\mathrm{mXBP} 1$ upstream, $5^{\prime}$-CCT TGT GGT TGA GAA CCA GG-3'; mXBP1 downstream, $5^{\prime}$-CTA GAG GCT TGG TGT ATA C-3'; hXBP1 upstream, 5' -CCT TGT AGT TGA GAA CCA GG-3'3 ; hXBP1 downstream, 5'-GGG GCT TGG TAT ATA TGT GG-3'. The PCR products were resolved by electrophoresis with an $8 \%$ polyacrylamide gel and were stained with ethidium bromide.

\section{Western Blotting}

Western blotting was performed as described previously (Hosoi et al., 2014) albeit with some modifications. Briefly, the cells were washed and lysed for 20 min with RIPA buffer containing $25 \mathrm{mM}$ Tris $\mathrm{HCl}$ ( $\mathrm{pH}$ 7.6), $150 \mathrm{mM} \mathrm{NaCl}, 1 \% \mathrm{NP}-40,0.1 \%$ SDS, $1 \%$ sodium deoxycholate, $1 \mathrm{mM} \mathrm{Na} \mathrm{VO}_{4}, 10 \mathrm{mM} \mathrm{NaF}, 10 \mu \mathrm{g} / \mathrm{mL}$ aprotinin, $10 \mu \mathrm{g} / \mathrm{mL}$ leupeptin, and $1 \mathrm{mM}$ phenylmethylsulfonyl fluoride (PMSF). The lysates were incubated on ice for $20 \mathrm{~min}$. Then, the samples were sonicated for $4 \mathrm{~min}$ and suspended for $30 \mathrm{~min}$ at $4^{\circ} \mathrm{C}$. After additional sonication for $4 \mathrm{~min}$, the samples were centrifuged at $14,000 \mathrm{rpm}$ for $5 \mathrm{~min}$ at $4^{\circ} \mathrm{C}$, and the supernatants were collected. The samples were then boiled in SDS sample buffer for $3 \mathrm{~min}$, fractionated with SDS-PAGE, and transferred to nitrocellulose membranes. These membranes were then incubated with anti-CD63 (Novus; 1:1000), anticalnexin (Enzo; 1:1000), anti-CD81 (Cell signaling; 1:1000), and anti-IRE1 $\alpha$ (Cell signaling; 1:1000) antibodies, followed by an anti-horseradish peroxidase-linked antibody. Peroxidase binding was detected using chemiluminescence with an enhanced chemiluminescence system.

\section{Collection of Exosomes}

Exosomes were collected from the cell culture medium $24 \mathrm{~h}$ after replacement with fresh medium containing exosome depleted FCS. ExoQuick-TC was used to collect the exosomes from the culture supernatant by following the manufacturer's instructions. The quality of the exosomes was checked by Western blotting with anti-CD63, anti-calnexin, and anti-CD81 antibodies.

\section{Electron Microscope Analysis}

Exosomes obtained were analyzed by scanning electron microscopy (SEM). The isolated samples were loaded on electron microscopy grid and then fixed with $2 \%$ glutaraldehyde and $2 \%$ paraformaldehyde for $1.5 \mathrm{~h}$ at room temperature. After washing with PBS, the samples were stained with $2 \%$ phosphotungstic acid solution $(\mathrm{pH} 7)$ for $20 \mathrm{~min}$ at room temperature. Then, the samples were dehydrated with ethanol $(50,70,80,90,95$, $100 \%$ ), incubated with tert-butyl alcohol, and dried using freeze dryer. After the dehydration, samples were coated with osmium tetroxide and analyzed with Field Emission Scanning Electron Microscope (JSM-7800F, JEOL Ltd.).

\section{Statistics}

Results are expressed as the mean \pm SEM. Statistical analyses were performed using Student's $t$-test (for Figure 1C) or Bonferroni-Dunn test (for Figure 2D).

\section{RESULTS}

\section{sXBP1 mRNA in Cells Was Incorporated Into the Exosomes}

We first collected the exosomes from MIN6 pancreatic $\beta$ cells and measured the levels of exosome marker of pancreatic cells, CD81 (Guay et al., 2015). In addition to whole cell lysates of cells, we observed the expression of CD81 in the exosomes of MIN6 pancreatic $\beta$ cells (Figure 1A). We did not detect calnexin in MIN6 pancreatic $\beta$ cells exosomes, which is known to be expressed only in the cells (Figure 1A). Furthermore, we detected exosome by electron microscope analysis (Figure 1B). These results suggest that the exosomes were efficiently collected and the extract of the exosome may not contaminate detectable levels of cellular debris. The sXBP1 transcript is expressed in high levels in pancreatic cells (Iwawaki et al., 2004), and sXBP1 is constitutively expressed in pancreatic $\beta$ cells (Engin et al., 2014). We observed the expression of mRNAs belonging to sXBP1 and an unspliced form of XBP1 (uXBP1) in MIN6 pancreatic $\beta$ cells as assed by RT-PCR (Figure 1C, left band of the panel). We then 
A

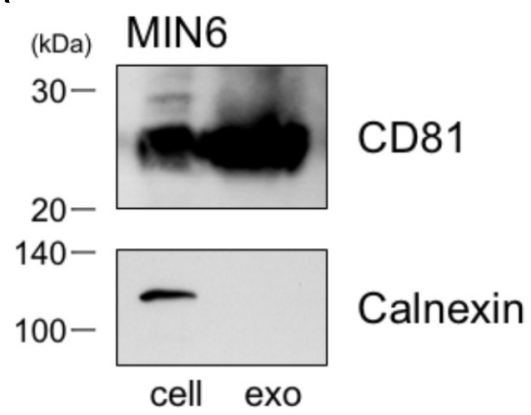

B

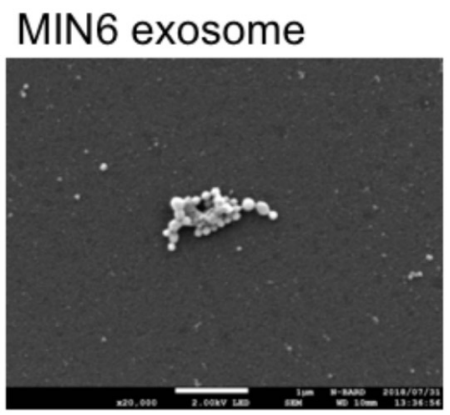

C

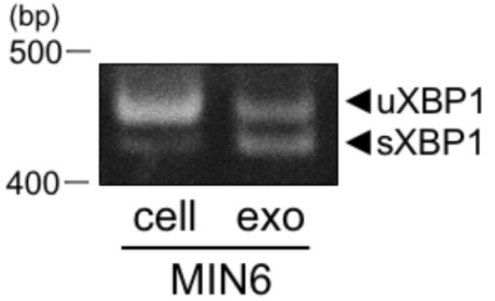

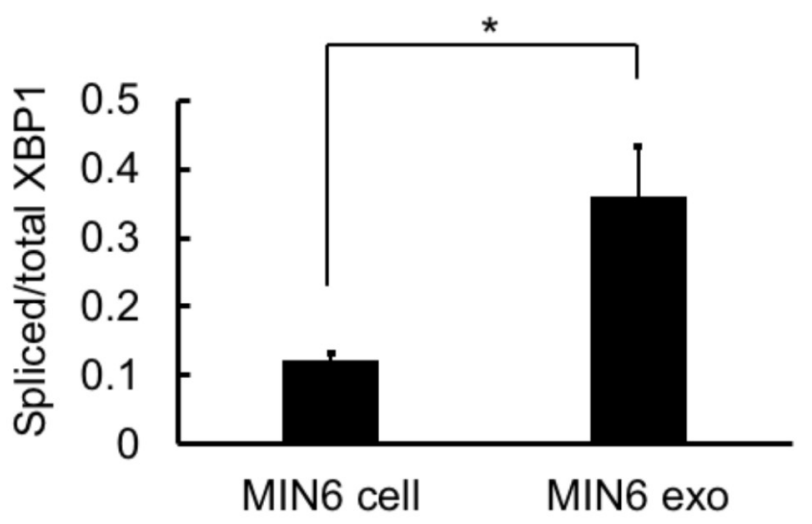

FIGURE 1 | Spliced form of XBP1 mRNA was incorporated into the exosomes of pancreatic $\beta$ cells. (A) Exosomes in the MIN6 cell culture supernatant expressed CD81 but not calnexin. MIN6 pancreatic $\beta$ cells were cultured for $24 \mathrm{~h}$ in DMEM containing exosome-depleted FCS. Then, the exosomes were extracted from the supernatant, and CD81 and calnexin levels were measured by Western blotting. Typical data of 3 independent experiments was shown. Other 2 data were shown in the Supplementary Figure 1A. (B) Electron microscope analysis of the exosomes obtained from MIN6 pancreatic $\beta$ cells. Scale bar: $1 \mu \mathrm{M}$. (C) MIN6 pancreatic $\beta$ cells were cultured for $24 \mathrm{~h}$ in DMEM containing exosome-depleted-FCS. Then, the exosomes were extracted from the supernatant. The mRNA levels of total and spliced forms of XBP1 (SXBP1) were analyzed in cells (left band of the panel) and exosomes (right band of the panel) by RT-PCR, with specific primers for XBP1. Typical data of 4 independent experiments was shown. Other 3 data were shown in the Supplementary Figure 1B. ${ }^{*} P<0.05$ by Student's $t$-test.

checked whether XBP1 mRNA in the cells was incorporated into the exosomes of MIN6 pancreatic $\beta$ cells. We performed RT-PCR analysis with specific primers for XBP1, and found that sXBP1 as well as $\mathrm{uXBP} 1$ were expressed in the exosomes (Figure 1C, right band of the panel). The levels of spliced/total XBP1 mRNA in the exosomes was higher than that of cells (Figure 1C). This suggests that sXBP1 mRNA was incorporated into the exosomes.

\section{sXBP1 mRNA in the Cells Was Incorporated Into the Exosome of HEK293T Cell, Which Overexpress IRE1 $\alpha$}

We collected the exosomes from the cellular supernatant and analyzed the expression of exosome marker, CD63. We observed CD63 expression in whole cell lysates of cells and in the exosomes of HEK293T cells (Figure 2A). We did not detect calnexin in the exosomes of HEK293T cells, which is known to be expressed only in the cells (Figure 2A). Thus, the result indicates no contamination of cellular compartment in the exosome. Furthermore, we detected exosome by electron microscope analysis (Figure 2B). These results suggest that the exosomes were efficiently collected and the extract of the exosome may not contaminate detectable levels of cellular debris. We then checked whether XBP1 mRNA in the cells was incorporated into the exosomes of IRE1 $\alpha$ overexpressed cells, as IRE1 $\alpha$ overexpression increases XBP1 splicing through its autophosphorylation (Tirasophon et al., 2000). IRE1 $\alpha$ construct was transfected into HEK293T cells and overexpression of IRE1 $\alpha$ in the cells was confirmed by Western blotting (Figure 2C). We also confirmed the increase of sXBP1 mRNA levels in the cells at IRE1 $\alpha$ overexpressed HEK293T cells by RT-PCR analysis (Figure 2D, left panel). We then analyzed the mRNA levels of uXBP1 and sXBP1 in the exosomes of these cells. As shown in Figure 2D, we observed higher levels of spliced/total XBP1 mRNA in the exosomes of HEK293T cells, both in mock- and IRE1 $\alpha$-transfected cells, than those in cells (Figure 2D, right panel). These results suggest that sXBP1 mRNA in the cells was incorporated into the exosomes. In addition, measurement of the levels of $\mathrm{UXBP} 1$ and sXBP1 indicated that the sXBP1 mRNA may have been incorporated in the exosomes of HEK293T cells (Figure 2D).

\section{DISCUSSION}

sXBP1 acts as a transcription factor, regulating cellular homeostasis by coping with stress (Yoshida et al., 2001). In the present study, we found that XBP1 and sXBP1 mRNAs were both expressed in the exosomes. We also found that the levels of spliced/total XBP1 mRNA in the exosomes were higher 
A

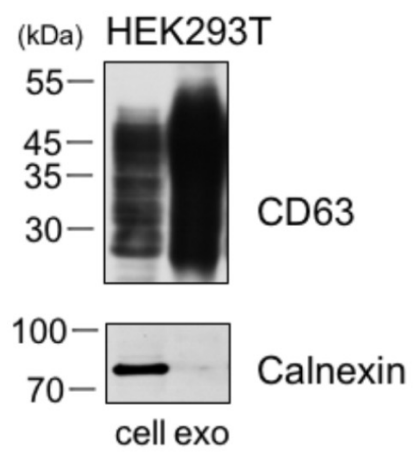

B

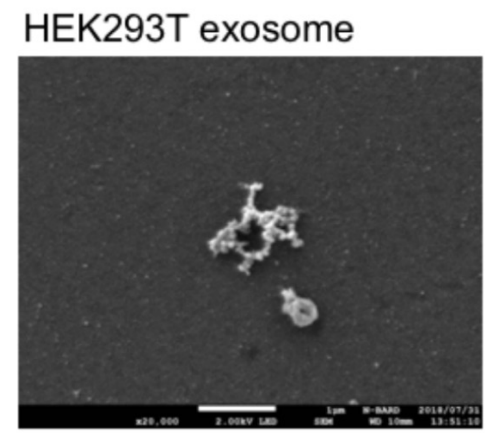

C
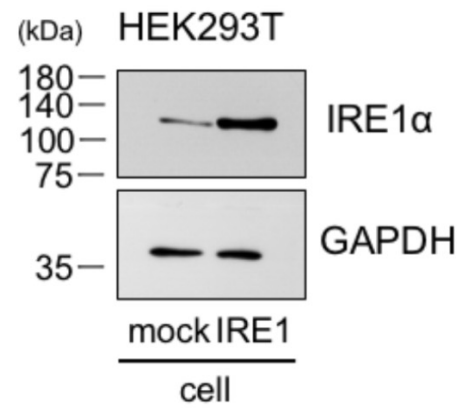

D
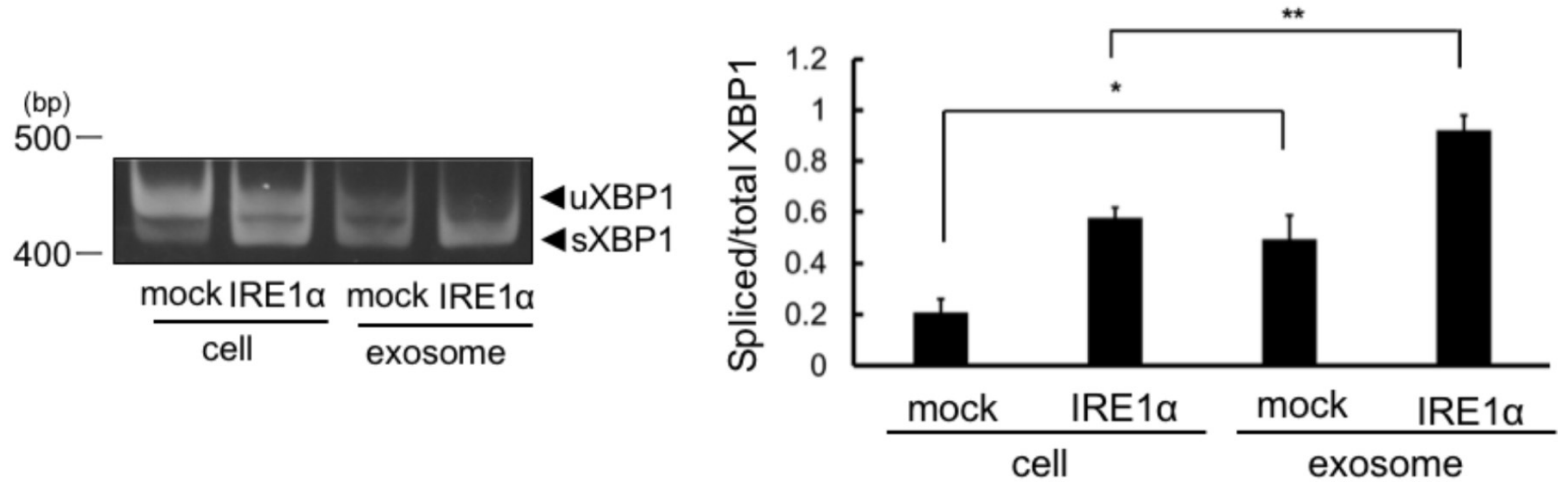

FIGURE 2 | Spliced form of XBP1 mRNA was incorporated into the exosomes of HEK293T cells, which overexpress IRE1 $\alpha$. (A) HEK293T cells were cultured for $24 \mathrm{~h}$ in DMEM containing exosome-depleted FCS. Then, the exosomes were extracted from the supernatant using ExoQuick-TC. CD63 and calnexin levels were measured by Western blotting. Typical data of 3 independent experiments was shown. Other 2 data were shown in the Supplementary Figure 2A. (B) Electron microscope analysis of the exosomes obtained from HEK293T cells. Scale bar: $1 \mu$ M. (C) HEK293T cells were transfected with Flag-IRE1 $\alpha$ construct and cultured in DMEM containing exosome-depleted FCS for $24 \mathrm{~h}$. IRE1 $\alpha$ overexpression in the cell was confirmed by Western blotting. (D) The exosomes were extracted from the supernatant, and the mRNA levels of total and spliced forms of XBP1 (sXBP1) were analyzed in cells and exosomes by RT-PCR, with specific primers for XBP1 (UXBP1, unspliced XBP1; sXBP1, spliced XBP1). Typical data of 5 independent experiments was shown. Other 4 data were shown in the Supplementary Figure 2B. ${ }^{* *} P<0.01$; ${ }^{*} P<0.05$ by Bonferroni-Dunn test.

when compared with its levels inside the cells. To date, the mechanisms involved in the incorporation of sXBP1 mRNA into the exosomes remain unelucidated. It has been reported that the formation of multivesicular bodies (MVBs) increases by ER stress (Kanemoto et al., 2016). Because the production of MVBs and their fusion with the plasma membranes (PM) plays a key role in the release of exosomes (van Niel et al., 2006), incorporation of sXBP1 mRNA would likely occur during this process. Currently, we do not know the molecular mechanisms of the incorporation of sXBP1 mRNA in the exosomes, which would be interesting future subjects. IRE1 $\alpha$ was suggested to play a role in releasing exosomes by ER stress (Kanemoto et al., 2016). Therefore, it would also be an interesting subject to analyze the molecular mechanisms how IRE $1 \alpha-X B P 1$ pathway was involved in releasing exosomes as well as incorporating sXBP1 mRNA in the exosome.

sXBP1 transcripts are highly expressed in pancreatic cells (Iwawaki et al., 2004) (Figure 1). In addition, exosomes can cross the blood-brain barrier (Alvarez-Erviti et al., 2011; Chen et al., 2016), a fact that could be exploited in treating neurodegenerative diseases (Jarmalavièiūtë and Pivoriûnas, 2016). Epidemiological evidence suggests that type 2 diabetes is a risk factor in the development of AD (Leibson et al., 1997; Pasinetti and Eberstein,
2008), and pancreatic $\beta$ cell function may be closely linked to $\mathrm{AD}$ onset. If pancreatic exosomes are absorbed by neurons, then they could be key to regulating neuronal function. sXBP1 was suggested to be involved in protecting amyloid $\beta$-induced neurotoxicity in Alzheimer's disease (AD) models (Casas-Tinto et al., 2011). Therefore, it would be an interesting future topic to investigate possible involvement of sXBP1 in the exosome in AD progression.

The manner in which ER stress signals are transmitted extracellularly remains unexplored. We found that one of the ER stress signal-induced transcripts, sXBP1, was incorporated into the exosomes. Our results suggest that exosomes may play a vital role in the extracellular release of ER stress signals. To our knowledge, this is the first report on incorporation of ER stress signal in the exosomes, and our results provide novel insights into the exosomal transmission of intercellular signal.

\section{AUTHOR CONTRIBUTIONS}

MN performed the experiment. TH and MN analyzed the data. $\mathrm{TH}$ conceived hypothesis, interpreted the data, and wrote the manuscript. KO discussed and checked the manuscript. 


\section{FUNDING}

This work was supported by the JSPS KAKENHI and Takeda Science Foundation.

\section{ACKNOWLEDGMENTS}

We thank Dr. David Ron (University of Cambridge) for kindly providing us the IRE1 $\alpha$ plasmid. Electron microscope analysis was performed by Field Emission Scanning Electron Microscope at Hiroshima University of Imaging Platform

\section{REFERENCES}

Alvarez-Erviti, L., Seow, Y., Yin, H., Betts, C., Lakhal, S., and Wood, M. J. (2011). Delivery of siRNA to the mouse brain by systemic injection of targeted exosomes. Nat. Biotechnol. 29, 341-345. doi: 10.1038/nbt. 1807

Calfon, M., Zeng, H., Urano, F., Till, J. H., Hubbard, S. R., Harding, H. P., et al. (2002). IRE1 couples endoplasmic reticulum load to secretory capacity by processing the XBP-1 mRNA. Nature 415, 92-96. doi: 10.1038/415 092a

Casas-Tinto, S., Zhang, Y., Sanchez-Garcia, J., Gomez-Velazquez, M., RinconLimas, D. E., and Fernandez-Funez, P. (2011). The ER stress factor XBP1s prevents amyloid-beta neurotoxicity. Hum. Mol. Genet. 20, 2144-2160. doi: 10.1093/hmg/ddr100

Chen, C. C., Liu, L., Ma, F., Wong, C. W., Guo, X. E., Chacko, J. V., et al. (2016). Elucidation of exosome migration across the blood-brain barrier model in vitro. Cell. Mol. Bioeng. 9, 509-529. doi: 10.1007/s12195-016-0458-3

Engin, F., Nguyen, T., Yermalovich, A., and Hotamisligil, G. S. (2014). Aberrant islet unfolded protein response in type 2 diabetes. Sci. Rep. 4:4054. doi: 10.1038/ srep04054

Guay, C., Menoud, V., Rome, S., and Regazzi, R. (2015). Horizontal transfer of exosomal microRNAs transduce apoptotic signals between pancreatic betacells. Cell Commun. Signal. 13:17. doi: 10.1186/s12964-015-0097-97

Hetz, C. (2012). The unfolded protein response: controlling cell fate decisions under ER stress and beyond. Nat. Rev. Mol. Cell Biol. 13, 89-102. doi: 10.1038/ nrm 3270

Hosoi, T., Suyama, Y., Kayano, T., and Ozawa, K. (2016). Flurbiprofen ameliorates glucose deprivation-induced leptin resistance. Front. Pharmacol. 7:354. doi: 10.3389/fphar.2016.00354

Hosoi, T., Yamaguchi, R., Noji, K., Matsuo, S., Baba, S., Toyoda, K., et al. (2014). Flurbiprofen ameliorated obesity by attenuating leptin resistance induced by endoplasmic reticulum stress. EMBO Mol. Med. 6, 335-346. doi: 10.1002/ emmm.201303227

Iwawaki, T., Akai, R., Kohno, K., and Miura, M. (2004). A transgenic mouse model for monitoring endoplasmic reticulum stress. Nat Med. 10, 98-102. doi: $10.1038 / \mathrm{nm} 1004-1013$

Jarmalavičiūté, A., and Pivoriūnas, A. (2016). Exosomes as a potential novel therapeutic tools against neurodegenerative diseases. Pharmacol. Res. 113(Pt B), 816-822. doi: 10.1016/j.phrs.2016.02.002

Kanemoto, S., Nitani, R., Murakami, T., Kaneko, M., Asada, R., Matsuhisa, K., et al. (2016). Multivesicular body formation enhancement and exosome release during endoplasmic reticulum stress. Biochem. Biophys. Res. Commun. 480, 166-172. doi: 10.1016/j.bbrc.2016.10.019 supported by the Ministry of Education, Culture, Sports, Science and Technology (MEXT), Japan. We appreciate Dr. Misako Imai-Takemoto, Ms. Kyoko Ikeda, Dr. Jun-ichi Kakimura for their kind support at the analysis of Electron microscope.

\section{SUPPLEMENTARY MATERIAL}

The Supplementary Material for this article can be found online at: https://www.frontiersin.org/articles/10.3389/fphys. 2018.01357/full\#supplementary-material

Leibson, C. L., Rocca, W. A., Hanson, V. A., Cha, R., Kokmen, E., O’brien, P. C., et al. (1997). Risk of dementia among persons with diabetes mellitus: a population-based cohort study. Am. J. Epidemiol. 145, 301-308. doi: 10.1111/j. 1749-6632.1997.tb48496.x

Pasinetti, G. M., and Eberstein, J. A. (2008). Metabolic syndrome and the role of dietary lifestyles in Alzheimer's disease. J. Neurochem. 106, 1503-1514. doi: 10.1111/j.1471-4159.2008.05454.x

Ron, D., and Walter, P. (2007). Signal integration in the endoplasmic reticulum unfolded protein response. Nat. Rev. Mol. Cell Biol. 8, 519-529. doi: 10.1038/ nrm2199

Schorey, J. S., Cheng, Y., Singh, P. P., and Smith, V. L. (2015). Exosomes and other extracellular vesicles in host-pathogen interactions. EMBO Rep. 16, 24-43. doi: 10.15252/embr.201439363

Tirasophon, W., Lee, K., Callaghan, B., Welihinda, A., and Kaufman, R. J. (2000). The endoribonuclease activity of mammalian IRE1 autoregulates its mRNA and is required for the unfolded protein response. Genes Dev. 14, 2725-2736. doi: $10.1038 /$ ncb1596

Valadi, H., Ekström, K., Bossios, A., Sjöstrand, M., Lee, J. J., and Lötvall, J. O. (2007). Exosome-mediated transfer of mRNAs and microRNAs is a novel mechanism of genetic exchange between cells. Nat. Cell Biol. 9, 654-659. doi: 10.1093/jb/ mvj128

van Niel, G., Porto-Carreiro, I., Simoes, S., and Raposo, G. (2006). Exosomes: a common pathway for a specialized function. J. Biochem. 140, 13-21. doi: $10.1093 / \mathrm{jb} / \mathrm{mvj} 128$

Walter, P., and Ron, D. (2011). The unfolded protein response: from stress pathway to homeostatic regulation. Science 334, 1081-1086. doi: 10.1126/ science. 1209038

Yoshida, H., Matsui, T., Yamamoto, A., Okada, T., and Mori, K. (2001). XBP1 mRNA is induced by ATF6 and spliced by IRE1 in response to ER stress to produce a highly active transcription factor. Cell 107, 881-891. doi: 10.1016/ S0092-8674(01)00611-0

Conflict of Interest Statement: The authors declare that the research was conducted in the absence of any commercial or financial relationships that could be construed as a potential conflict of interest.

Copyright (c) 2018 Hosoi, Nakashima and Ozawa. This is an open-access article distributed under the terms of the Creative Commons Attribution License (CC BY). The use, distribution or reproduction in other forums is permitted, provided the original author(s) and the copyright owner(s) are credited and that the original publication in this journal is cited, in accordance with accepted academic practice. No use, distribution or reproduction is permitted which does not comply with these terms. 